\title{
A LOGNORMAL RELIABILITY DESIGN MODEL
}

\author{
Edward. E. Osakue', Lucky Anetor ${ }^{2}$ \\ ${ }^{1}$ Department of Industrial Technology, Texas Southern University, Houston, Texas, USA \\ ${ }^{2}$ Department of Mechanical Engineering, Nigerian Defense Academy, Kaduna, Nigeria
}

\begin{abstract}
It is well known that design loads vary randomly during equipment operations. Similarly, material properties such as yield strength, tensile strength, fatigue strength, etc. are random variables. Design analytical models are approximations of reality and failure mode models are also approximations. Consequently, design solutions are not exact. Practical design must therefore, consider the random nature and statistical variability of design parameters. Reliability-based design models are developed to provide practical design methods. This paper develops a lognormal reliability-based design model that can be coded in Excel Spreedsheet. Two design examples are considered in demonstrating the application of the formulated model. In the first example, our result differs from the result from [7] by $0.9 \%$ on the conservative side. The weight of the beam in the second example differs from [9] by $14.53 \%$ positively for a reliability target of $z=3$. This variance is largely due to differences in reliability targets. When the beam size is adjusted to closely match the reliability levels of [9], the weight of the beam becomes $1.4 \%$ lower for our model. Therefore the results from our reliability model give comparable but slightly conservative and realistic solutions.
\end{abstract}

Keywords: Lognormal, Reliability, Variability, Normal Variate

\section{INTRODUCTION}

In engineering design, parameters such as loads, material properties, component geometry, manufacturing precision, service environment, and product usage are subject to variability $[1,2]$. In order to accommodate variability in design parameters and analytical models, probabilistic design methods are used. When quality, reliability, and safety are paramount, probabilistic design becomes the preferred method desired [3]. According to Wang [1], reliability-based design is the most robust and economical design when design parameter statistics are well defined. When means and or standard deviations of design parameters have uncertainties, reliability-based robust design is useful in minimizing the impact of such uncertainties.

The use of probabilistic design methods requires some appropriate probability density distribution [4]. Commonly used probability density distributions in mechanical and structural designs are normal, lognormal, and Weibull distributions. The beta and Gumbel (type 1 extreme value) distributions are occasionally applied. However, the Weibull, beta, and Gumbel distribution functions do not lend themselves to closed form solutions like the normal or lognormal distributions. Design models generally have sums and products of the relevant parameters. The sum of variates from any distribution tends asymptotically to approach normal and the sum of normal variates is normal. The products and quotients of lognormal variates are lognormal. Real powers of a lognormal variate are themselves lognormal. Products of variates from any distribution tend asymptotically to approach lognormal. The multiplication of variates that have normal distribution yields results that tend to be lognormal in distribution [5]. The lognormal probability density function is appropriate for random quantities representing the product of independent random variables and like the normal probability density function; it is applicable to the sum of random variables.

Hess et al. [6] suggested that the lognormal probability density function is appropriate for yield strength for steels used in ship building. A chi-square goodness of fit test at $95 \%$ confidence level on a histogram of 1000 ultimate tensile strength tests of 1020 steel did not reject the null hypothesis of lognormality [5]. Fatigue strength data are very often described with lognormal probability density function [4, 7]. The lognormal distributions are frequently used for material properties and fatigue lives [8]. A lognormal probability density function may be assumed for the Young's modulus on the basis of its limitation to nonnegative values [6]. Similarly, the Poisson's ratio may be associated with the lognormal probability density function. Castillo et al. [9] based their design study of a crane on the mixed frequency distributions of Gumbel and lognormal for design parameters. Lu, He, and Zhang [10] used the lognormal probability density function for the robust design of an arbor. Lognormal probability density function is typically adopted for load variable in the analysis of ship structural reliability or random variables that always take positive values [11]. Histograms of data from many manufacturing processes exhibit the "bell-shaped" pattern of the normal distribution [12]. Component and assembly dimensions are positive numbers, so the lognormal probability density function is equally an appropriate choice for dimensional variables.

The lognormal probability density function can be used where the normal probability density function is used. It is somewhat more accurate compared to the normal probability density function [13]. From the cited references above, it may be concluded that the lognormal probability density 
function is, in most cases, a good approximation for design capacity models, mechanical strengths or capabilities, load, and component dimensions. Therefore, from the perspective of accuracy and universality, the lognormal probability density function appears to be a strong candidate of choice for probabilistic design and more attractive than the normal probability density function.

Most probability density function can be summarized by dominant parameters and the most common parameters are the mean and variance or standard deviation. The cov or $\mathrm{COV}$ is the ratio of standard deviation to the mean value. It is a dimensionless parameter and is a particularly useful measure of uncertainty because it can be used to summarize the variability of a group of materials and equipment in an industrial sector $[13,14]$. A small uncertainty would typically be represented by a cov of 0.05 while a considerable uncertainty would be indicated by a cov of 0.25 [15]. A variable with a zero value cov reduces to a deterministic parameter. Thus the cov may be considered as a quality parameter and could be used in the selection of material vendors or suppliers in some other design and manufacturing business transactions

Deterministic design traditionally uses a safety or design factor to accommodate uncertainties in design parameters. A safety factor is subjectively assigned in practice so this approach does not provide a logical basis for addressing uncertainties or variability. With the safety factor method, the level of reliability cannot be assessed quantitatively; therefore the safety margin in a design is practically unknown. Hence this approach can lead to over-design and under-design issues. The globalization of the economy and technology and the need to assure consumer safety are rendering this approach uncompetitive.

The goal of this study is to develop a lognormal reliability design model that can be used in component design. Because the lognormal probability function provides closed form solutions and can be more accurate than the normal probability function, it can be potentially used in component design when high reliability is desired. The design parameters are assumed to have lognormal probability density function and the uncertainty associated with each design parameter is estimated using sensitivity analysis of the first order Taylor's series expansion. This approach reduces the usually difficult and expensive probabilistic and sensitivity analysis in reliability-based and optimizationbased designs into algebraic equations that can be coded in Spreadsheet programs such as Microsoft Excel. The need for highly specialized skills and advanced mathematical analysis can, therefore, be minimized at the initial or preliminary design stages. Both the use of general purpose Spreadsheet programs and lower skilled design personnel can lead to cost savings in design projects.

\section{A LOGNORMAL RELIABILITY MODEL}

The lognormal reliability model is summarized in this section. A detail presentation of the development of the model is in the Appendix. Please refer to it if in doubt of any relationship summarized here.
Consider a capacity model of the form:

$$
\begin{aligned}
& C_{M}=f\left(x_{1}, x_{2}, x_{3}, \ldots\right) \\
& \mu_{C M}=f\left(\mu_{x 1}, \mu_{x 2}, \mu_{x 3}, \ldots\right)
\end{aligned}
$$

When design capacity models are evaluated using mean values of significant parameters, the expected result has a reliability of $50 \%$, which corresponds to unit normal variate of 0 . Increased reliability needs higher values of the unit normal variate.

The standard deviation of $\mu_{C M}$ can be estimated using sensitivity analysis of the first order Taylor's series expansion. This approximately given by:

$$
s_{C M}=\sqrt{\sum_{i=1}^{n}\left(\frac{\partial C_{M}}{\partial x_{i}}\right)^{2} s_{x_{i}}^{2}}
$$

The cov of $\mu_{C M}$ is:

$$
\vartheta_{C M}=\frac{s_{C M}}{\mu_{C M}}
$$

Now consider a random variable that is a sum of some independent random variables. That is:

$$
C_{M}=f\left(x_{1}+x_{2}+x_{3} \ldots\right)
$$

Applying the rules of Eqs. (2), (3), and (4) to Eq. (5), we have:

$$
\begin{aligned}
& \mu_{C M}=f\left(\mu_{x 1}+\mu_{x 2}+\mu_{x 3} \ldots .\right) \\
& \vartheta_{C M}=\frac{1}{\mu_{C M}} \sqrt{\vartheta_{x 1}^{2}+\vartheta_{x 2}^{2}+\vartheta_{x 3}^{2}+\ldots}
\end{aligned}
$$

Next consider a random variable that is a product of independent random variables:

$$
C_{M}=f\left(x_{1}^{a} x_{2}^{b} x_{3}^{c} \ldots .\right)
$$

Applying the rules of Eqs. (2), (3), and (4) to Eq. (6), we have:

$$
\begin{aligned}
& \mu_{C M}=f\left(\mu_{x 1}^{a} \mu_{x 2}^{b} \mu_{x 3}^{c} \ldots . .\right) \\
& \vartheta_{C M}=\sqrt{a^{2} \vartheta_{x 1}^{2}+b^{2} \vartheta_{x 2}^{2}+c^{2} \vartheta_{x 3}^{2}+\ldots .}
\end{aligned}
$$

Specific forms of relationship between design parameters in Eq. (2) are dependent on the type of failure under consideration. Most practical design capacity models generally consist of a combination of Eq. (6a) and Eq. (8a). 
Successive application of Eqs. (6b) and (8b) can help in deducing the covs of design capacity models by inspection.

Every component in an assembly or sub-assembly has a limit in its ability to resist imposed load. This limit is here called "mechanical capability" which is the maximum load capacity of component. Load is construed in generic term that may mean axial force load, lateral force load, bending moment load, and twisting moment (torsional) load. Design loads can be determined by constructing load diagrams such as axial force diagram, shear force diagram, bending moment diagrams, etc. A mechanical capability may be the yield strength, tensile strength, fatigue strength, critical lateral deflection, critical torsional deformation, critical axial deformation, critical frequency, etc. of a component.

Suppose we associate $C_{M}$ with a mechanical capability $C_{F}$ that has statistical parameters $\mu_{C F}$ and $\vartheta_{C F}$ as mean and cov, respectively. We may define a random capability ratio as:

$$
n=\frac{C_{F}}{C_{M}}
$$

The reliability factor is defined as:

$$
n_{z}=\frac{\mu_{C F}}{\mu_{C M}}
$$

When $C_{F}$ and $C_{M}$ are assumed to have lognormal distributions, $n$ will have a lognormal distribution too. In the Appendix, a conservative standard deviation of $n$ is proposed and from Eq. (A7):

$$
\left.s_{z}=\sqrt{\operatorname{In}\left\{\left(1+\vartheta_{C M}^{2}\right)\left(1+\vartheta_{C F}^{2}\right)\right.}\right\}
$$

Failure of a component occurs if $n \leq 1$ and the unit normal variate for failure is from Eq. (A11):

$$
z=\frac{\operatorname{In}\left(n_{z}\right)-0.5 s_{z}^{2}}{s_{z}}
$$

The design reliability for $\mathrm{z} \geq 1.28$ or $R_{z} \geq 90 \%$ is:

$$
R_{z}=1-10^{-\left(0.2006 z^{2}+0.2299 z+0.3818\right)}
$$

\section{DESIGN PARAMETERS VARIABILITY}

The proposed reliability design model uses the mean and cov values of design parameters. Mean vales of design parameters are much easier to generate than cov values, since data on covs have not been generally documented. Design capacity models are subject to variability due to assumptions made in their formulations and so are approximations of reality. Simplified models in engineering design are generally sufficiently accurate and may deviate from reality by about $\pm 10 \%$ [16]. Assuming the error spans 4 standard deviations, this approximates to a coefficient of variation of $5 \%$ for machine design. In structural design, typical values of cov for analytical model uncertainties are 3 $-15 \%$ [17].

Variability in service load is usually the largest in most design problems but most difficult to predict, especially at the design phase [18]. The user may not follow guidelines; service environment may be different from that assumed during design, and in fact, a host of variables completely outside the control of the designer come into play. The cov of rated load may be in the range of 3 to $20 \%$ [19]. Estimations of dead weights of building materials based on tabulated data or reported in codes are prone to error due differences between data values in tables and those of material manufacturers. Therefore estimate of dead loads in buildings during preliminary design can be in error by $15 \%$ to $20 \%$ [20].

Failure mode models for uniaxial tension, uniaxial compression, biaxial stress state, triaxial stress state, fatigue damage, etc. are applied in the design of many components. These failure models have varying degrees of accuracy but variability information about them is generally lacking. If uniaxial stress state failure is the case, then accurate failure theories are available and Ullman [21] suggest a cov of $2 \%$ where they are applicable. For a static biaxial stress failure mode, such as combined bending and shear stresses, a cov of $5 \%$ is assumed here. Shear yield strength failure prediction from tensile yield strength has a cov of $3 \%$ for steel materials [7].

Allowable lateral deflection is commonly based on experience or the deformation that may be tolerated by supporting bearings. Variation obviously exists but quantification does not seem to exist at this time. Using judgment, a cov of $5 \%$ to $10 \%$ is suggested, the lower values being for machine design and higher values for structural designs. Geometric parameter variability is generally small: the cov for mating dimensions in mechanical components is about $0.1 \%$ [5], cov of principal dimension for standard structural shape is about $1.5 \%$, cov of principal dimension for fabricated structural shape is about $2 \%$, and component length dimension has a cov in the range of 0.1 to $0.3 \%$.

\section{DESIGN SIZING AND VERIFICATION}

The design analysis of a component generally involves iteration in two basic tasks: design sizing and design verification as depicted in Fig. 1. The task in design sizing is determining the form and size of a member for a desired reliability level. The form of a member is defined by its length and cross-sectional shape and dimensions over its length. In general, the cross-section may vary along the length of a member but this makes analysis more complicated and costly. Components with constant crosssectional shapes are usually the first choice, especially 
during preliminary design but modifications often occur later in the design process.

The length of a member is often based on space limitation and may be estimated in a preliminary design layout diagram but can be refined later, perhaps from rigidity or strength considerations. The cross-section can be sized for an assumed shape based on yield strength, fatigue strength or other serviceability criteria. Design sizing involves use of suitable serviceability criteria such as strength, transverse deflection, torsional deformation, buckling, etc. along with the type of load and its configuration in the estimation of the dimensions of components or assembly.

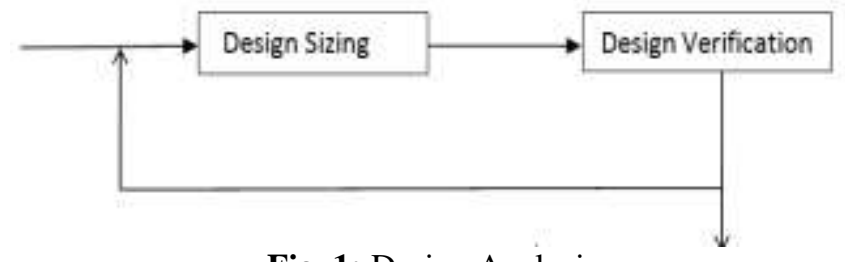

Fig. 1: Design Analysis

In some design situations such as fatigue design, $\vartheta_{C M}$ cannot be accurately evaluated until dimensions of a component are known due to the influence of stress concentration. In such cases, an approximate value $\vartheta_{C O}$ of $\vartheta_{C M}$ may be used in Eq. (11) to obtain an approximate lognormal standard deviation. That is:

$$
\left.s_{z o}=\sqrt{\operatorname{In}\left\{\left(1+\vartheta_{C O}^{2}\right)\left(1+\vartheta_{C F}^{2}\right)\right.}\right\}
$$

From Eq. (12) a design factor $n_{o}$ that is statistically based may be estimated as:

$$
n_{o}=\exp \left[s_{z o}\left(z_{o}+0.5 s_{z o}\right)\right]
$$

Geometric properties of cross-sectional shape such as area, section modulus, and second moment of area (area inertia) can be reduced to be a function of one dimension through proportional design. Hence it may be generalized that:

$$
\mu_{g}=f\left(\mu_{L}, \mu_{G}, \mu_{P}, \mu_{C F}, n_{o}\right)
$$

Design verification deals with the assessment of the adequacy of a component or assembly design on the basis of a desired reliability target. In deterministic design approach, the safety factor or design factor is used for design adequacy assessment. Design verification is done to ensure that the selected form and dimensions of a component or assembly meet design requirements. A design is accepted as adequate if the evaluated expected reliability level is at least equal to a desired target. A factor of reliability greater than unity is necessary for failure avoidance.

Generally in machine and structural designs, design capacity models are functions of load and component geometry. That is:

$$
\mu_{C M}=f\left(\mu_{g}, \mu_{L}, \mu_{G}, \mu_{P}\right)
$$

Formal acceptance of design adequacy is established by use of Eqs. (10) and (12). For acceptable design:

$$
n_{z}=\frac{\mu_{C F}}{\mu_{C M}} \geq n_{o} \quad z \geq z_{o}
$$

The requirement of $n_{z} \geq n_{o}$ satisfies traditional design adequacy based on "safety factor" and $z \geq z_{o}$ satisfies a reliability target value.

\section{DESIGN EXAMPLES}

In order to demonstrate how to use the reliability model developed, two design examples are considered next. The first example is very simple, being chosen to clarify the concept and application of the reliability model for design sizing and verification tasks. The second example extends the application of the reliability model by considering several failure modes.

\subsection{Example 1: Design Sizing and Verification}

A cold drawn steel round bar with a yield strength 540(1, $0.074) \mathrm{MPa}$ is subjected to a static force $220(1,0.082) \mathrm{kN}$. (a) What is the diameter of the bar if a reliability of $99.9 \%$ $\left(z_{o}=3.09\right)$ is desired? (b) Estimate the design reliability for a chosen diameter size [7].

\subsubsection{Solution for Example 1}

a) Design sizing requires a numeric value for the design factor. This is evaluated from the target reliability and design capacity model variability. The tensile stress capacity model gives the average tensile stress in the bar and is:

$$
\sigma_{t}=\frac{4 F_{o}}{\pi d^{2}}
$$

Using the rules of Eq. (8), by inspection of Eq. (18):

$$
\begin{aligned}
& \vartheta_{\sigma}^{2}=\vartheta_{s f}^{2}+4 \vartheta_{d}^{2} \\
& \vartheta_{C M}^{2}=\vartheta_{\sigma}^{2}+\vartheta_{m a}^{2}
\end{aligned}
$$

The, applied static load is considered so no stress concentration factor is required. Since design capacity model is that of uni-axial stress, accuracy is known to be high, so model accuracy cov of $3 \%$ will be assumed. The design load has a cov given as 0.082 . The geometric parameter cov will be neglected in this example. Without stress concentration factor, $\vartheta_{s k}$ is zero in Eq. (A15). The adjustment model accuracy $\operatorname{cov} \vartheta_{s a}$, is zero, as well as the miscellaneous cov, $\vartheta_{s m}$. Thus Eq. (A15) reduces to: 


$$
\vartheta_{s f}=\vartheta_{s o}
$$

The yield strength cov is given as 0.074. No adjustment in the tensile yield strength of the rod is necessary, assuming the rod is used in ordinary environment. Hence in Eq. (A16) from the Appendix, the value $C_{f_{s}}$ is unity and $\vartheta_{f c}$ is zero in Eq. (A17). The miscellaneous cov is zero and Eq. (A17) reduces to:

$$
\begin{aligned}
& \vartheta_{C F}=\sqrt{\vartheta_{y t}^{2}+\vartheta_{f a}^{2}} \\
& \vartheta_{f o}=\vartheta_{y t}=0.074
\end{aligned}
$$

Uni-axial tensile failure is expected and the failure model is well understood, so a variability of $2 \%$ [21] is assumed. That is:

$$
\vartheta_{f a}=0.02
$$

The design input data are summarized in Table 1.

Table 1: Input Data for Example 1

\begin{tabular}{|l|l|}
\hline Capacity model accuracy cov & $\vartheta_{m a}=0.03$ \\
\hline Design load cov & $\vartheta_{s f}=0.082$ \\
\hline Rod diameter cov & $\vartheta_{d}=0$ \\
\hline Yield strength cov & $\vartheta_{y t}=0.074$ \\
\hline Failure mode model accuracy cov & $\vartheta_{f c}=0.02$ \\
\hline Design load (N) & $F_{o}=220 \times 10^{3}$ \\
\hline Tensile yield strength (MPa) & $S_{y t}=540$ \\
\hline
\end{tabular}

For design sizing, Eq. (18) is modified as:

$$
\sigma_{t}=\frac{4 F_{o}}{\pi d^{2}} \leq \frac{S_{y t}}{n_{o}}
$$

And:

$$
d \geq \sqrt{\frac{4 n_{o} F_{o}}{\pi S_{y t}}}
$$

Eqs. (19b), (20), and (14) are used to obtain $s_{z o}$. Eq. (15) is used to $n_{o}$ and Eq. (22) is used to estimate the rod diameter. (b) For a chosen diameter size, the expected stress is obtained from Eq. (18) and the reliability factor is obtained from Eq. (10). Finally the unit normal variate and reliability level are obtained from Eq. (12) and and (13) respectively. Table 2 summarizes the computational results for design example 1.
Table 2: Results for Example 1

\begin{tabular}{|l|l|}
\hline Estimated diameter size (mm) & 27.34 \\
\hline Diameter size of [7] (mm) & 27.10 \\
\hline Chosen diameter size (mm) & 28.0 \\
\hline Design factor & 1.441 \\
\hline Reliability factor & 1.513 \\
\hline Reliability level (\%) & 99.98 \\
\hline Reliability level (\%) of [7] & 99.92 \\
\hline
\end{tabular}

The diameter design size $d=28 \mathrm{~mm}$ is acceptable since the estimated $R_{z}=99.98 \%$ for a desired value of $99.9 \%$ is acceptable. The solutions for this problem in [7, p. $251-$ 252] are $d=27.1 \mathrm{~mm}$ and $R_{z}=99.92 \%(z=3.157)$. This design example considers only tensile failure mode but the next example involves other failure modes such as shear and bending.

\subsection{Design Example 2}

Fig. 1 shows an overhead travelling crane (OTC) with a single bridge girder. The crane consists of a bridge girder mounted on two end trucks that run on runway girders. The bridge girder is perpendicular to the runway girders and supports a trolley which carries the hoist. The hoist is used to lift the load vertically [22] Fig 2 shows four possible modes of failure [9] of the girder and it is desired to design it as a plate girder as shown in Fig. 3. The load on the girder is $600 \mathrm{kN}$ and the allowable maximum deflection is $l / 888$, where $l$ is beam span which is $6 \mathrm{~m}$. The plates for the gilder construction are from steel material with yield strength

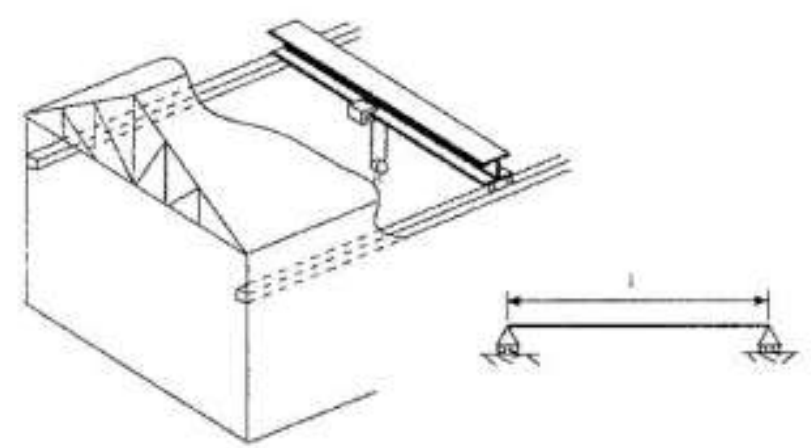

Fig. 1: Bridge crane [9]
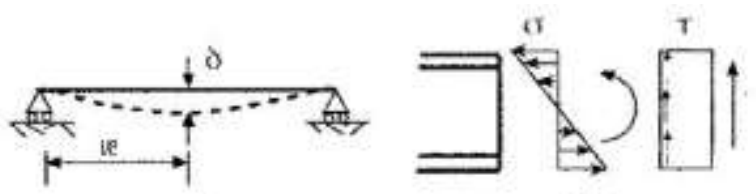

(a)

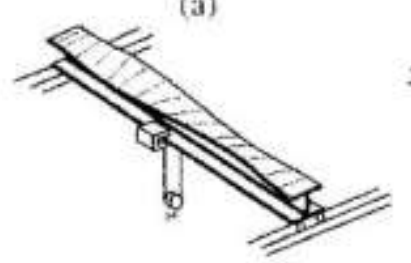

(b)

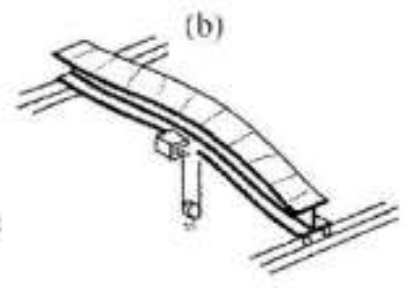

(c)

(d)

Fig.2: Crane girder failure modes [9] 
of $395 \mathrm{MPa}$. Design the girder assuming a minimum reliability goal of 3.0 standard deviations; subject to $8 \mathrm{~mm}$ $\leq t_{w} \leq 38 \mathrm{~mm}, 8 \mathrm{~mm} \leq t_{f} \leq 38 \mathrm{~mm}$, and $b_{f} \geq 300 \mathrm{~mm}$ Consider the design parameters as random variables with coefficient of variation $1 \%$ for the geometric parameters shown in Fig. 3 The coefficient of variation for yield strength of steels is $7 \%$ and that of the Young's modulus is 3\% [9].

\subsection{Solution Analysis}

In providing solution to design problem 2 , it is assumed that the crane bridge girder is loaded by a combination of concentrated and distributed loads. The concentrated load comes from the weight of the load being hoisted, the weight of the hoist and the weight of the trolley. The distributed load comes from the weight of the plate girder. Plate girders are built-up beams used when standard rolled products are not adequate. The depth of the girder ranges from one-fourteenth to one-sixth [23] of the span depending on configuration. Web depth-web thickness ratio and width-flange thickness ratio (see Fig. 3) are limited depending on specific design conditions; recommendations are available from American Institute of Steel Construction (AISC).

\subsection{Flange Geometric Properties}

Fig. 3 shows the dimensional parameters of a plate girder. The dimensions can be related proportionally.

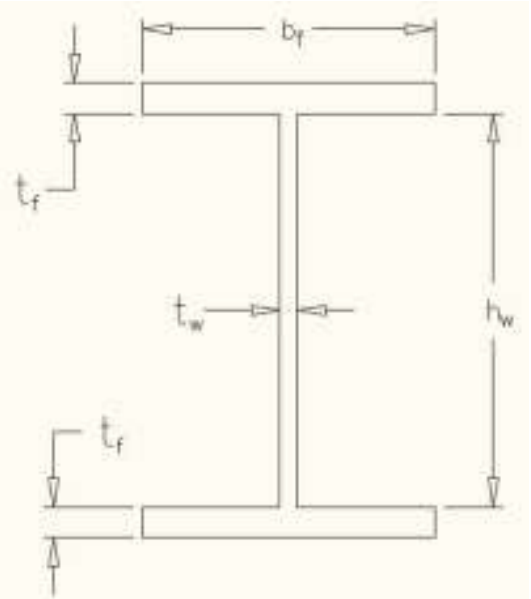

Fig. 3: Girder section parameters

Referring to Fig. 3, and assuming proportional design:

$$
\begin{aligned}
& \alpha=\frac{b_{f}}{t_{f}} \leq \alpha_{c} \\
& \beta=\frac{h_{w}}{t_{w}} \leq \beta_{c} \\
& \gamma=\frac{h_{w}}{b_{f}}
\end{aligned}
$$

Based on AISC recommendations [23] and from [9]:

$$
\begin{aligned}
& \alpha_{c}=30 \sqrt{\frac{276}{S_{Y}}} \\
& \beta_{c}=750 \sqrt{\frac{16565}{S_{Y}\left(S_{Y}+114\right)}}
\end{aligned}
$$

Eqn. 24a is valid for unstiffened web. For a failure mode in initial design, it is assumed that:

$$
\alpha_{o}=\frac{\alpha_{c}}{n_{o}} \quad \beta_{o}=\frac{\beta_{c}}{n_{o}}
$$

The area inertia $I_{x}$ for the girder is [9]:

$$
I_{x}=\frac{1}{12}\left[b_{f}\left(h_{w}+2 t_{f}\right)^{3}-\left(b_{f}-t_{w}\right) h_{w}^{3}\right]
$$

Substitute Eq. (23) in Eq. (26):

$$
I_{x}=\frac{\varpi_{I}}{12} h_{w}^{4}
$$

where:

$$
\varpi_{I}=\frac{1}{\gamma}\left(1+\frac{2}{\alpha \gamma}\right)^{3}-\left(\frac{1}{\gamma}-\frac{1}{\beta}\right)
$$

The section modulus $Z_{x}$ is:

$$
Z_{x}=\frac{2 I_{x}}{h}=\frac{\varpi_{Z}}{6} h_{w}^{3}
$$

where:

$$
\varpi_{z}=\frac{1}{\gamma}\left(1+\frac{2}{\alpha \gamma}\right)^{2}-\frac{\left(\frac{1}{\gamma}-\frac{1}{\beta}\right)}{\left(1+\frac{2}{\alpha \gamma}\right)}
$$

Referring to Fig. 3:

$$
\begin{aligned}
& h=2 t_{f}+h_{w} \\
& A_{f}=t_{f} b_{f} \\
& A_{w}=t_{w} h_{w}
\end{aligned}
$$




$$
\begin{aligned}
& A_{b}=2 A_{f}+A_{w} \\
& q_{o}=\rho g A_{b} \\
& W_{o}=q_{o} l
\end{aligned}
$$

\subsection{Flange Bending Stress Design}

The lower surface of the bottom flange or the upper surface of the top flange experience tensile and compressive bending stresses. The bending stress is:

$$
\begin{aligned}
& \sigma_{b}=\frac{M_{x}}{Z_{x}} \\
& M_{x}=K_{m c} F_{o} l+K_{m d} W_{o} l
\end{aligned}
$$

Applying the rules of Eqs. (6) and (8) by inspection to Eqs. (33) and (34), we have:

$\vartheta_{b}=\frac{\sqrt{\vartheta_{s f}^{2}+\vartheta_{l}^{2}+\vartheta_{Z}^{2}+\eta_{\sigma}^{2}\left(\vartheta_{q}^{2}+\vartheta_{l}^{2}+\vartheta_{Z}^{2}\right)}}{1+\eta_{\sigma}}$

$\eta_{b}=\frac{K_{m d} W_{o}}{K_{m c} F_{o}}$

The cov of dead weight will be taken as $5 \%\left(\vartheta_{q}=0.05\right)$ [20]. The crane load is described as being the maximum, so no service factor is necessary. From the Appendix, Eq. (A15) reduces to:

$$
\vartheta_{s f}=\vartheta_{s o}
$$

And:

$$
\vartheta_{C M}=\sqrt{\vartheta_{b}^{2}+\vartheta_{m a}^{2}}
$$

No adjustment in tensile yield strength is necessary, assuming crane is used in ordinary environment. Hence in Eq. (A16) from the Appendix, the value $C_{f s}$ is unity and $\vartheta_{f c}$ is zero in Eq. (A17). The miscellaneous cov is non-zero due to combination of normal and shear stress based on distortion energy theory and Eq. (A17) reduces to:

$$
\vartheta_{C F}=\sqrt{\vartheta_{y t}^{2}+\vartheta_{f a}^{2}+\vartheta_{f m}^{2}}
$$

With values from Eqs. (36b) and (36c); Eqs. (11), (12) and (13) are used for reliability assessment of the flange for bending stress failure.
For design sizing the bending stress should not exceed the yield strength of the component material divided by the design factor. That is:

$$
\frac{M_{x}}{Z_{x}} \leq \frac{S_{y t}}{n_{o}}
$$

From Eq. (35b), the weight of the bridge girder is required before $\vartheta_{b}$ can be determined. However, the cross-sectional dimensions of the beam are unknown at initial design stage in the design process, so an approximate value of $\vartheta_{b}\left(\vartheta_{b o}\right)$ is necessary in order to estimate the design factor, $n_{o}$. By assuming a load-beam weight ratio, $\vartheta_{b o}$ can be determined. Therefore:

$$
\vartheta_{C O}=\sqrt{\vartheta_{b o}^{2}+\vartheta_{m a}^{2}}
$$

Using evaluated values from Eq. (36c) and Eq. (38) in Eq. (15), $n_{o}$ can be estimated. Substitute Eq. (28a) in Eq. (37), and transform into:

$$
h_{w} \geq\left(\frac{6 n_{o} M_{x}}{\varpi_{Z} S_{y t}}\right)^{\frac{1}{3}}
$$

\subsection{Beam Transverse Deflection Design}

The beam deflection is estimated as:

$$
\begin{aligned}
& \delta=\frac{l^{3}}{E I_{x}}\left[K_{c \delta} F_{o}+K_{d \delta} W_{o}\right] \\
& n_{z}=\frac{\delta_{A}}{\delta}
\end{aligned}
$$

Applying the rules of Eqs. (6) and (8) by inspection to Eq. (40a), we obtain:

$$
\begin{aligned}
\vartheta_{\delta}^{2}= & \frac{\vartheta_{s f}^{2}+\vartheta_{E}^{2}+9 \vartheta_{l}^{2}+\vartheta_{I}^{2}}{\left(1+\eta_{\delta}\right)^{2}} \\
& +\frac{\eta_{\delta}^{2}\left(\vartheta_{q}^{2}+\vartheta_{E}^{2}+16 \vartheta_{l}^{2}+\vartheta_{I}^{2}\right)}{\left(1+\eta_{\delta}\right)^{2}}
\end{aligned}
$$

Where

$$
\begin{gathered}
\eta_{\delta}=\frac{K_{d \delta} W_{o}}{K_{c \delta} F_{o}} \\
\vartheta_{C M}=\sqrt{\vartheta_{\delta}^{2}+\vartheta_{m a}^{2}}
\end{gathered}
$$


No adjustment in allowable deflection. From the Appendix, Eq. (A19) reduces to:

$$
\vartheta_{C F}=\sqrt{\vartheta_{A}^{2}+\vartheta_{f a}^{2}}
$$

With values from Eqs. (42a) and (42b); Eqs. (11), (12) and (13) are used for reliability assessment for deflection failure.

For design sizing:

$$
\begin{aligned}
& \frac{1}{E I_{x}}\left[K_{c \delta} F_{o} l^{3}+K_{d \delta} W_{o} l^{3}\right] \leq \frac{\delta_{A}}{n_{o}} \\
& \delta_{A}=\frac{l}{888}
\end{aligned}
$$

From Eq. (41b), the weight of the bridge girder is required before $\vartheta_{b}$ can be determined. However, the cross-sectional dimensions of the beam are unknown at this point in the design process, so an approximate value of $\vartheta_{\delta}\left(\vartheta_{\delta o}\right)$ is necessary in order to estimate the design factor, $n_{o}$. By assuming a load-beam weight ratio, $\vartheta_{\delta o}$ can be determined. Therefore:

$$
\vartheta_{C O}=\sqrt{\vartheta_{\delta o}^{2}+\vartheta_{m a}^{2}}
$$

Using evaluated values from Eq. (42a) and Eq. (43c) in Eq. (15), $n_{o}$ can be estimated. Substitute Eq. (27a) in Eq. (43a) and rearrange to obtain:

$$
h_{w} \geq\left[\frac{12 \times 888 \times n_{o} l^{2}}{\varpi_{I} E}\left[K_{c \delta} F_{o}+K_{d \delta} W_{o}\right]\right]^{1 / 4}
$$

\subsection{Beam Global Buckling Design}

Lateral instability in thin deep beam is possible in plate girders and can be assessed through the critical buckling moment [9]. This moment may be obtained as:

$$
\begin{aligned}
& M_{c r}=\frac{\kappa_{s} E}{l \sqrt{2(1+v)}} \\
& n_{z}=\frac{M_{c r}}{M_{x}}
\end{aligned}
$$

where:

$$
I_{s}=\sqrt{I_{y} I_{t}}
$$

and:

$$
I_{y}=\frac{1}{12}\left[2 t_{f} b_{f}^{3}+h_{w} t_{w}^{3}\right]
$$

$$
I_{t}=\frac{1}{3}\left[2 t_{f}^{3} b_{f}+h_{w} t_{w}^{3}\right]
$$

Neglecting the cov of Poisson' ratio, apply the rules of Eq (8) by inspection to Eq. (45a):

$$
\vartheta_{c r} \approx\left[\vartheta_{E}^{2}+\vartheta_{l}^{2}+\vartheta_{I}^{2}\right]^{1 / 2}
$$

The cov of $M_{x}$ in Eq. (45b) is obtained from Eqn. (34) which is given by Eq. (35a). That is:

$$
\vartheta_{M_{x}}=\vartheta_{b}
$$

Then:

$$
\vartheta_{C M}=\sqrt{\vartheta_{b}^{2}+\vartheta_{m a}^{2}}
$$

No adjustment in tensile elastic modulus is necessary, assuming the crane is used in ordinary environment. From the Appendix, Eq. (A17) reduces to:

$$
\vartheta_{C F}=\sqrt{\vartheta_{c r}^{2}+\vartheta_{f a}^{2}}
$$

With values from Eqs. (49a) and (49b); Eqs. (11), (12) and (13) are used for reliability assessment of the beam for buckling failure.

\subsection{Combined Bending and Shear Stresses}

The upper surface of the bottom flange or the lower surface of the top flange experience both bending and shear stresses. The combined tensile stress on the flanges can be evaluated and checked based on distortion energy failure of ductile materials:

$$
\begin{aligned}
& \sigma_{t}=\sqrt{\sigma_{b}^{2}+3 \tau_{s}^{2}} \\
& n_{z}=\frac{S_{y t}}{\sigma_{t}} \\
& \sigma_{b}=\frac{K_{m c} F_{o} l}{Z_{x}^{\prime}}+\frac{K_{m d} W_{o} l}{Z_{x}^{\prime}} \\
& \tau_{s}=\frac{K_{s c} F_{o}}{A}+\frac{K_{s d} W_{o}}{A} \\
& Z_{x}^{\prime}=\frac{h^{h}}{h_{w}} Z_{x}
\end{aligned}
$$

Apply the rules of Eqs. (3) and (4) to Eq. (50a): 


$$
\begin{aligned}
& \vartheta_{t}=\frac{1}{1+3 \eta_{s}^{2}}\left[\vartheta_{b}^{2}+9 \eta_{s}^{4} \vartheta_{\tau}^{2}\right]^{\frac{1}{2}} \\
& \eta_{s}=\frac{\tau_{s}}{\sigma_{b}}
\end{aligned}
$$

Then:

$$
\vartheta_{C M}=\sqrt{\vartheta_{t}^{2}+\vartheta_{m a}^{2}}
$$

Apply the rules of Eqs. (6) and (8) by inspection to Eq. (51b):

$$
\begin{aligned}
\vartheta_{\tau}^{2}= & \frac{\vartheta_{s f}^{2}+\vartheta_{A}^{2}}{\left(1+\eta_{\tau}\right)^{2}} \\
& +\frac{\eta_{\tau}^{2}\left(\vartheta_{q}^{2}+\vartheta_{A}^{2}+\vartheta_{l}^{2}\right)}{\left(1+\eta_{\tau}\right)^{2}} \\
\eta_{\tau}= & \frac{W_{o}}{F_{o}}
\end{aligned}
$$

The prediction of shear yield strength from tensile yield strength based on deformation energy theory for ductile materials introduces a miscellaneous cov in the mechanical capability of the beam. Thus from the Appendix, Eq. (A17) reduces to:

$$
\vartheta_{C F}=\sqrt{\vartheta_{y t}^{2}+\vartheta_{f a}^{2}+\vartheta_{f m}^{2}}
$$

With values from Eqs. (53c) and (54c); Eqs. (11), (12) and (13) are used for reliability assessment for flange failure.

\subsection{Web Stress Design}

The web needs to be checked for shear failure. This is done using the applied load and the weight of the beam.

$$
\begin{aligned}
& \tau_{w}=\frac{1.15\left(K_{s c} F_{o}+K_{s d} W_{o}\right)}{A_{w}} \\
& n_{z}=\frac{S_{y t}}{\sqrt{3} \tau_{w}}
\end{aligned}
$$

The cov of the web shear stress is obatained as in Eq. (54a). Then:

$$
\vartheta_{C M}=\sqrt{\vartheta_{\tau w}^{2}+\vartheta_{m a}^{2}}
$$

No adjustment in tensile yield strength is necessary, assuming the crane is used in ordinary environment. However, the prediction of shear yield strength from tensile yield strength based on deformation energy theory for ductile materials introduces a miscellaneous cov in the mechanical capability of the beam. Thus from the Appendix, Eq. (A17) reduces to Eq. (54c). With values from Eqs. (56a) and (56b); Eqs. (11), (12) and (13) are used for reliability assessment of the web for shear stress failure.

\subsection{Solution Results for Example 2}

For a simply supported beam carrying a concentrated load, the maximum bending moments occurs at midspan $(l / 2)$ and $K_{m c}=1 / 4 ; \quad K_{s c}=1 / 2 ; \quad$ and $K_{c \delta}=1 / 48$. For uniformly distributed weight load, the maximum bending moment also occurs at mid span with $K_{m d}=1 / 8 ; K_{s d}=1 / 2$; and $K_{d \delta}=$ $5 / 384$. The value of $\kappa=3.1416$ [9] in Eqn. 24b. The coefficient of variation of the load is approximated as 0.117 from information in [9]. The Young modulus of steel is taken as $210 \times 10^{3} \mathrm{MPa}$. Eqns. (24) to (56) were coded into Excel spreadsheet and used for the design sizing and design verification of the plate girder. Two pages were developed: one for design sizing and the other for design verification.

Table 3: Design Sizing for Example 2

\begin{tabular}{|l|l|l|}
\hline \multirow{2}{*}{$\begin{array}{l}\text { Design } \\
\text { Parameter }\end{array}$} & $\begin{array}{l}\text { Estimate } \\
\text { Bending }\end{array}$ & $\begin{array}{l}\text { Lateral } \\
\text { Deflection }\end{array}$ \\
\hline Web Size \\
\hline$h_{w}(\mathrm{~mm})$ & 763.26 & 919.09 \\
\hline$t_{w}(\mathrm{~mm})$ & 5.80 & 7.00 \\
\hline$A_{w}\left(\mathrm{~mm}^{2}\right)$ & 4426.50 & 6418.33 \\
\hline Flange Size & \multicolumn{2}{|l}{} \\
\hline$b_{f}(\mathrm{~mm})$ & 254.42 & 306.36 \\
\hline$t_{f}\left(\mathrm{~mm}^{2}\right)$ & 16.57 & 19.95 \\
\hline$A_{f}\left(\mathrm{~mm}^{2}\right)$ & 4215.37 & 6112.23 \\
\hline Beam Size & & 959.00 \\
\hline$h(\mathrm{~mm})$ & 796.40 & 18642.80 \\
\hline$A_{b}\left(\mathrm{~mm}^{2}\right)$ & 12857.20 & 1435.50 \\
\hline$q_{o}(\mathrm{~N} / \mathrm{m})$ & 990.00 & 8612.97 \\
\hline$W_{o}(\mathrm{~N})$ & 5940.03 & \\
\hline
\end{tabular}

Table 3 shows the initial size estimates for the beam size based on flange bending stress and lateral deflection. The solution for the bending stress seems to be a lower bound case while that for deflection appears to be an upper bound case. Both solutions fall short of the required minimum size for the web thickness and flange width. Table 4 shows the design verification iterations. In iteration column 1 , the web thickness and flange width are forced at their minimum required values while the web depth is reduced. Four iterations yielded the chosen solution on iteration column 4 for a minimum of $z=3$. The iteration column 5 is for the solution from reference [9]. Iteration 6 is done for the purpose of comparing the reliability model being presented with that of reference [9]. 
Table 4: Beam design iterations for Example 2

\begin{tabular}{|c|c|c|c|c|c|c|}
\hline \multirow[b]{2}{*}{ Design Parameters } & \multicolumn{4}{|c|}{ Iteration } & \multicolumn{2}{|c|}{ Comparison } \\
\hline & 1 & 2 & 3 & 4 & {$[9]$} & Current \\
\hline$h_{w}(\mathrm{~mm})$ & 750 & 750 & 750 & 735 & 726.10 & 730 \\
\hline$t_{w}(\mathrm{~mm})$ & 8 & 10 & 10 & 10 & 8 & 8 \\
\hline$b_{f}(\mathrm{~mm})$ & 300 & 310 & 310 & 310 & 355.9 & 330 \\
\hline$t_{f}(\mathrm{~mm})$ & 20 & 25 & 30 & 30 & 23.44 & 25 \\
\hline$h_{(\mathrm{mm})}$ & 790 & 800 & 810 & 795 & 773.44 & 780 \\
\hline$W_{o}(\mathrm{~N})$ & 8316.0 & 10626.0 & 12058.2 & 11988.8 & 10467.58 & 10321.01 \\
\hline $\mathrm{z}$ (Flange bending) & 5.200 & 6.804 & 7.827 & 7.682 & 6.886 & 6.801 \\
\hline $\mathrm{z}$ (Flange combined stress) & 4.831 & 6.525 & 7.511 & 7.362 & 6.325 & 6.269 \\
\hline $\mathrm{z}$ (Lateral deflection) & 0.409 & 2.188 & 3.356 & 3.075 & 2.045 & 2.014 \\
\hline $\mathrm{z}$ (Lateral buckling) & -0.502 & 2.937 & 5.295 & 5.292 & 3.943 & 3.651 \\
\hline $\mathrm{z}$ (Web shearing) & 9.016 & 10.363 & 10.361 & 10.239 & 8.815 & 8.848 \\
\hline
\end{tabular}

In Castillo et al [9], the "reliability index" was used for design adequacy assessment. The reliability indices for combined stress, lateral deflection, lateral buckling and web shear stress are respectively $4.277,1.700,3.100$, and 6.832 for the optimum solution. Wang [1] indicated that the "safety or reliability index" parameter is problem dependent and often controversial. Since the unit normal variate is not problem dependent and non-controversial, it was felt that it might be informative to evaluate the unit normal variate based on the beam size solutions of [9] using the reliability model in our approach. The respective unit normal variates obtained for combined stress, lateral deflection, lateral buckling and web shear stress are respectively $6.325,2.045$, 3.943, and 8.815. Clearly, there are noticeable differences between the "reliability index" and unit normal variate values, so it is concluded that the "reliability index" is not equal to or is the same as the unit normal variate.

\section{SUMMARY}

In design Example 1, the diameter value in our solution is $27.34 \mathrm{~mm}$ while that of [7] is $27.1 \mathrm{~mm}$. The result from our model differs from the cited reference value by $0.9 \%$ on the conservative side. Thus the reliability model being presented appears slightly conservative but realistic. This problem is a simple one but helps to illustrate how the formulated model can be applied logically and systematically in more complex situation such as design example 2 .

Design Example 2 solution is shown in iteration 4 in Table 4. In this solution, $\alpha=10.33$ and $\beta=73.5$, and they are less than the respective critical values, which according to AISC recommendation, are $\alpha_{c}=25.08$ and $\beta_{c}=215.3$ for the steel strength of $395 \mathrm{MPa}$. The beam depth-span ratio is 0.123 , and it is in the range of 0.071 to 0.167 for plate girders [23]. Therefore, the solution obtained in this study seems quite acceptable. The beam weight for our solution is $14.53 \%$ higher than that of [9] for minimum value of $z=3$. However, when the beam size is adjusted to approximate the reliability levels of [9] as shown in the current column of Table 4, the beam weight is $1.4 \%$ lower. The beam sizes in the two comparison columns of Table 4 indicate very close agreement between our model results and those of [9].
It has been that realistic design solutions can be achieved by using a probabilistically obtained "safety factor" which is called "design factor", an approximation of the "reliability factor". The design or reliability factor depends on two parameters: coefficient of variation and quality or reliability goal specified in units of standard deviation. Based on the good agreement between the results from our model and the cited references, the lognormal distribution is deemed satisfactory in the probabilistic design of mechanical and structural components. Design adequacy in reliability-based design should be assessed based on the unit normal variate. That is, the quality goal requirement should always be used for design verification in probability-based design unless when relevant codes and or regulations suggest otherwise. It is concluded that the unit normal variate and "reliability or safety index" are not the same parameter or equal in design adequacy assessment. Probabilistic designs normally require special software and are sometimes very computationally intensive. This study shows, however, that reliability-based design can be performed with Excel: Microsoft Spreedsheet program, providing opportunities for cost reductions in design projects.

\section{CONCLUSION}

A lognormal reliability-based model has been presented. The model considers design parameters as random variables and characterizes them with a mean value and a coefficient of variation (cov). The cov of each design parameter is estimated using sensitivity analysis of the first order Taylor's series expansion. The absolute values of design parameters are not required in the reliability model which is surprisingly simple because it is a function of only two parameters: the reliability parameter $(\mathrm{z})$ and the variability parameter $\left(s_{z}\right)$. The reliability parameter is the unit (standard) normal variate and defines the level of risk acceptable in a design task. The variability parameter is the lognormal standard deviation of the assumed lognormal probability density function. It combines all the significant uncertainties in the design capacity model into one value. The reliability and variability parameters define the reliability factor for a specific design. Thus, if the variability of the significant factors in a design 
model can be estimated with reasonable accuracy, it is possible to design to a risk level through a reliability factor. The design approach reduces the usually difficult and expensive probabilistic and sensitivity analysis in reliabilitybased and optimization-based designs into algebraic equations that can be coded in Spreadsheet programs such as Microsoft Excel. This offers a low-cost based solution to reliability-based design.

\section{REFERENCES}

[1] Wang, W., Wu, J., Lust, R. V., (1997), Deterministic Design, Reliability-Based Design and Robust Design. Retrieved from https://web.mscsoftware.com/support/library/conf/auc 97/p02597.pdf

[2] Avoiding Common Mistakes and Misapplications in Design for Reliability (DFR). Retrieved from http://www.reliasoft.com/newsletter/v12i1/systhesis.ht m.

[3] Safie, F. M. and Weldon, D., (2007), Design for Reliability and Safety Approach for the New NASA launch Vehicle, $2^{\text {nd }}$ IAASS Conference on Space Safety in a Global World, Chicago.

[4] Johnson, C. R. (1980), Optimum Design of Mechanical Elements, Wiley, New York.

[5] Shigley, J. E. and Mischke, C. R. (Chief Editors), (1996), Standard Handbook of Machine Design, McGraw-Hill, New York.

[6] Hess, P. E., Bruchman, D., Assakkat, I. A., and Ayyub, B. M. (2002), Uncertainties in Material Strength, Geometric, and Load Variables. Retrieved from http://www.assakkaf.com/papers/Journals.

[7] Budynas, R. G. \& Nisbett, J. K., Shigley's Mechanical Engineering Design, McGraw Hill Education, pp. 253, $330-347$

[8] Probabilistic Statistical Distributions Technical Background. Retrieved from https://www.efatigue.com/probabilistic/background/sta tdist.html

[9] Castillo, E., Conejo, A. J., Minguez, R. and Castillo, C., (2003), An alternative approach for addressing the failure probability-safety factor method with sensitivity analysis, Reliability Engineering and System Safety, 82: $201-216$.

[10] Lu, H., He, Y. \& Zhang, Y. (2014), Reliability-Based Robust Design of Mechanical Components with Correlated Failure Modes Based on Moment method, Advances in Mechanical Engineering, http://dx.doi.org/10.1155/2014/568451.

[11] Paik, J. K. \& Frieze, P. A. (2001), Ship Structural Safety and Reliability, Prog. Struct. Engng Mater. Vol. 3, pp. $198-210$.

[12] Rufe, P. D. (editor), (2002), Fundamentals of Manufacturing, $2^{\text {nd }}$ ed., Society of manufacturing Engineers, Dearborn.

[13] Roshetov, D., Ivanov, A. and Fadeev, V. (1990), Reliability of Machines, MIR, Moscow, Chap. 4.

[14] Pandit, S. M. and Shiekh, A. K., (1980), Reliability and Optimal Replacement via Coefficient of Variation, Journal of Machine Design, Vol. 102, 761 -768.
[15] Factor of Safety and Probability of Failure. Retrieved from https://www.palisade.com/downloads/probability density

function/Factor_of_safety_and_probability_of_failure _with_@RISK.-probability density function.

[16] Matthews, C., (2005), ASME Engineer's Data Book, $2^{\text {nd }}$ ed. ASME Press.

[17] Faber, M. H. and Sorensen, J. D., (2002), Reliability Based Code Calibration, Joint Committee on Structural Safety, www.jcss.byg.dtu/.../FABER, Accessed 4-20-15.

[18] Wang, H., Kim, N. H., and Kim, Y., (2006), Safety Envelope for Load Tolerance and its Application to Fatigue Reliability, Journal of Mechanical Design, 128: 919 - 927.

[19] Osakue, E. E., (2015), Lognormal Reliability-Based Component Design, Technical Report, Dept. of Industrial Technologies, Texas Southern University, Houston, Texas.

[20] Hibbeler, R. C. (1999), Structural Analysis, $4^{\text {th }}$ ed. Prentice Hal, p. 11.

[21] Ullman, G. D, The Mechanical Design Process, $2^{\text {nd }}$ ed., McGraw-Hill, New York, Appendic C.

[22] Williams, A. (2011), Steel Structures Design, McGraw Hill, New York, p. 195 - 196.

[23] Johnson, B. G., Lin, F. J., and Galambos, T. V., (1986), Basic Steel design, $3^{\text {rd }}$ ed., Prentice-Hall, Upper Saddle River, New Jersey, p. 267.

\section{NOMENCLATURE}

$C_{M}=$ random variable of design capacity

model for a failure mode

$C_{F}=$ random variable of mechanical capability for a failure mode

$x_{i}=\mathrm{i}^{\text {th }}$ random design parameter

$f(\ldots)=$ function of

$s_{C M}=$ standard deviation of design capacity

model

$\frac{\partial C_{M}}{\partial x_{i}}=$ partial derivative of design capacity

model with respect to $i^{\text {th }}$ random

design parameter

$s_{x_{i}}=$ standard deviation of $\mathrm{i}^{\text {th }}$ random design

parameter

$n$ = capability ratio random variable for a

failure mode

$n_{z}=$ reliability factor

$s_{z}=$ standard deviation of $n$

$z=$ unit normal variate corresponding to failure

$R_{z}=$ design reliability at $\mathrm{z}$

$s_{z o}=$ approximate standard deviation of design capacity model

$n_{o}=$ design factor (approximate value of $n_{z}$ ) 
$z_{o}=$ unit normal variate for desired reliability level

$d=$ diameter of bar

$b_{f}=$ flange width

$t_{f}=$ flange thickness

$h_{w}=$ web height

$t_{w}=$ web thickness

$h$ = beam depth

$h_{o}=$ characteristic design dimension

$A_{f}=$ cross-sectional area of flange

$A_{w}=$ cross-sectional area of web

$A_{b}=$ cross-sectional area of beam

$q_{o}=$ weight per unit length of beam

$I_{x}=$ area inertia of beam about $\mathrm{x}$-axis

$M_{x}=$ maximum bending moment about x-axis

$Z_{x}=$ section modulus of beam about $\mathrm{x}$-axis

$Z_{x}^{\prime}=$ section modulus of beam about $\mathrm{x}$-axis at end of web

$g=$ acceleration due to gravity $\left(9.81 \mathrm{~m} / \mathrm{s}^{2}\right)$

$E=$ tensile elastic modulus

$K_{c \delta}=$ concentrated load deflection factor

$K_{d \delta}=$ distributed load deflection factor

$M_{c r}=$ critical buckling moment

$M_{x}=$ maximum bending moment

$I_{s}=$ stability or buckling area inertia

$I_{y}=$ area inertia about y-axis

$I_{t}=$ area inertia for twisting resistance

$K_{s c}=$ concentrated load direct shear factor

$K_{s d}=$ distributed load direct shear factor

$K_{m c}=$ concentrated load moment factor

$K_{m d}=$ distributed load moment factor

$l=$ length of beam

$W_{o}=$ weight of beam

$S_{y t}=$ mean yield strength of component material

$K_{s}=$ service load factor

$K_{s o}=$ rated load overload or external

dynamic load factor

$K_{s k}=$ stress concentration factor

$C_{z}=$ size factor

$C_{r}=$ surface roughness factor

$C_{t}=$ temperature factor

$s_{t}=$ standard deviation value of $t$

$t=$ lognormal variable of $n$

$t_{f}=$ normal variate corresponding to a failure probability $z_{f}=$ unit or standard normal variate corresponding to a failure probability

$n=$ capability-load ratio random variable

$\alpha=$ flange width-thickness ratio

$\alpha_{c}=$ critical flange width-thickness ratio

$\alpha_{o}=$ initia design value for $\alpha$

$\beta=$ web height-thickness ratio

$\beta_{c}=$ critical web height-thickness ratio

$\beta_{o}=$ initia design value for $\beta$

$\gamma=$ web height-flange width ratio

$\varpi_{I}=$ area inertia factor

$\varpi_{Z}=$ section modulus factor

$\rho=$ mass density of beam

$\kappa=$ critical buckling factor

$v=$ Poisson's ratio

$\sigma_{t}=$ expected tensile stress

$\delta=$ maximum deflection of beam

$\delta_{A}=$ maximum allowable deflection for component design

$\eta_{\sigma}=$ bending moment weight-load ratio

$\eta_{\sigma}=$ deflection weight-load ratio

$\tau_{s}=$ direct shear stress

$\eta_{s}=$ shear stress ratio

$\tau_{w}=$ web shear stress

$\eta_{\tau}=$ shear stress weight-load ratio

$\sigma_{b}=$ maximum bending stress

$\tau_{s}=$ direct shear stress

$\tau_{w}=$ web shear stress

$\mu_{x i}=$ mean value of $\mathrm{i}^{\text {th }}$ random design parameter

$\mu_{L}=$ mean value of generic independent design load

$\mu_{G}=$ mean value of generic independent geometric parameter

$\mu_{P}=$ mean value of generic independent material property parameter

$\mu_{g}=$ generic dependent geometric design parameter

$\mu_{C M}=$ mean value of design capacity model

$\mu_{C F}=$ mean adjusted value of mechanical capability

$s_{C F}=$ standard deviation value of mechanical capability

$s_{C M}=$ standard deviation of design capacity model

$\vartheta_{C M}=$ cov of design capacity model

$\vartheta_{C F}=$ cov of mechanical capability

$\vartheta_{f o}=$ cov of nominal mechanical capability value

$\vartheta_{f a}=$ cov of failure mode model accuracy 
$\vartheta_{f m}=$ cov of miscellaneous effects on capability

$\vartheta_{f c}=$ cov for mechanical capability correction

$\vartheta_{c t}=\operatorname{cov}$ of temperature factor

$\vartheta_{c z}=$ cov of size factor

$\vartheta_{c r}=$ cov of surface roughness factor

$\vartheta_{s f}=$ cov of service load

$\vartheta_{s o}=$ cov of rated load or external dynamic load

factor

$\vartheta_{s a}=\operatorname{cov}$ of service load factor model accuracy

$\vartheta_{s m}=$ cov of miscellaneous effects on service load

$\vartheta_{s k}=$ cov of stress concentration factor

$\vartheta_{m a}=$ cov of design model capacity accuracy

$\vartheta_{b}=$ cov of bending stress capacity model

$\vartheta_{\sigma}=$ cov of normal tensile stress load factor

$\vartheta_{d}=$ cov of bar diameter

$\vartheta_{F}=$ cov of design load

$\vartheta_{l}=$ cov of beam length

$\vartheta_{Z}=$ cov of beam section modulus

$\vartheta_{b o}=$ approximation of $\vartheta_{b}$

$\vartheta_{\delta}=$ cov of deflection capacity model

$\vartheta_{E}=$ cov of tensile elastic modulus

$\vartheta_{q}=$ cov of beam distributed weight

$\vartheta_{\delta o}=$ approximation of $\vartheta_{\delta}$

$\vartheta_{M_{x}}=$ cov of bending moment

$\vartheta_{c r}=$ cov of critical buckling moment

$\vartheta_{t}=$ cov of effective tensile stress

$\vartheta_{\tau}=$ cov of direct shear stress

$\vartheta_{\tau w}=\operatorname{cov}$ of $\tau_{w}$

$\mu_{n}=$ mean value of $n$

$\mu_{t}=$ mean value of $t$

$\vartheta_{n}=$ cov of capability ratio

\section{APPENDIX}

\section{A Lognormal Reliability Model Development}

This section explores the application of the lognormal probability distribution function in developing a design model for reliability factor. The lognormal probability distribution function has inherent properties that make it an attractive choice for engineering design applications. For instance, the products and quotients of lognormal variates are lognormal and real powers of lognormal variates are also lognormal [5]. Now most design formulas are products and sums of design parameters; so they can be assumed to have lognormal distribution as a first approximation. A lognormal random variable is a random variable whose natural logarithm has a normal distribution.

Suppose in a design model $C_{M}$ and $C_{F}$ represent applied load random variable and design model capability random variable, respectively, in the physical or linear domain. $C_{M}$ is associated with statistical parameters $\mu_{C M}, s_{C M}$, and $\vartheta_{C M}$. Likewise, $C_{F}$ is associated with statistical parameters $\mu_{F M}, s_{C F}$, and $\vartheta_{C F}$. Then:

$$
\begin{gathered}
\vartheta_{C M}=\frac{s_{C M}}{\mu_{C M}} \\
\vartheta_{C F}=\frac{s_{C F}}{\mu_{C F}}
\end{gathered}
$$

A random variable $n$ with parameters $\mu_{\mathrm{n}}$ and $\vartheta_{\mathrm{n}}$ can be defined as the ratio of $L_{M}$ and $C_{F}$. That is:

$$
\begin{aligned}
& n=\frac{C_{F}}{C_{M}} \\
& \vartheta_{n}=\sqrt{\vartheta_{C M}^{2}+\vartheta_{C F}^{2}}
\end{aligned}
$$

To preclude failure, $n$ must be greater than unity: that is, $C_{M}<C_{F}$. If $C_{M} \geq C_{F}$, then failure has occurred.

Assuming that $C_{F}$ and $C_{M}$ have lognormal distributions, respectively, then the quotient $n$, will be lognormal in distribution [24]. If $t$ is the random variable for the quotient $n$ in the lognormal domain, then:

$$
t=\ln (n)=\ln \left(\frac{C_{F}}{C_{M}}\right)
$$

Based on the properties of lognormal distribution, if the parameters of $t$ are $\mu_{t}$ and $s_{t}$, then:

$$
\begin{gathered}
\mu_{t}=\ln \left(\mu_{n}\right)-0.5 s_{t}^{2} \\
s_{t}=\sqrt{\left\{\operatorname{In}\left[1+\vartheta_{n}^{2}\right]\right\}} \\
=\sqrt{\left\{\operatorname{In}\left[1+\vartheta_{C M}^{2}+\vartheta_{C F}^{2}\right]\right\}}
\end{gathered}
$$

An engineering model can be deduced from Eq. (A6) if a slight modification on the expression for $s_{t}$ is introduced by changing it to the value predicted by the overlap in 
probability density functions of $L_{M}$ and $C_{F}$ [24]. This is given by:

$$
\begin{gathered}
s_{t}=\sqrt{\left\{\operatorname{In}\left[\left(1+\vartheta_{C F}^{2}\right)\left(1+\vartheta_{C M}^{2}\right)\right]\right\}} \\
=\sqrt{\left\{\operatorname{In}\left[1+\vartheta_{C F}^{2}+\vartheta_{C M}^{2}+\vartheta_{C F}^{2} \vartheta_{C M}^{2}\right]\right\}}
\end{gathered}
$$

Notice the additional product term $\left(\vartheta_{C F}^{2} \vartheta_{C M}^{2}\right)$ in Eqn. (A7) which is absent from Eq. (A6). This implies that the value of $s_{t}$ from Eq. (A7) will be slightly higher than that from Eq.
(A6). For small values of load and capability covs, results from Eqs. (A6) and (A7) will hardly be different. However, as values of the covs increase, then a difference will show up. At high reliability, this difference may be important.

The probability density distribution of $t$ is normal and is depicted in Fig. 1a. Fig. 1b depicts the corresponding unit normal variate distribution. The failure probability associated with the unit normal variate is represented by the area under the normal distribution curve (shaded in Fig. 1b) and corresponds to the failure region in Fig. 1a (shaded).

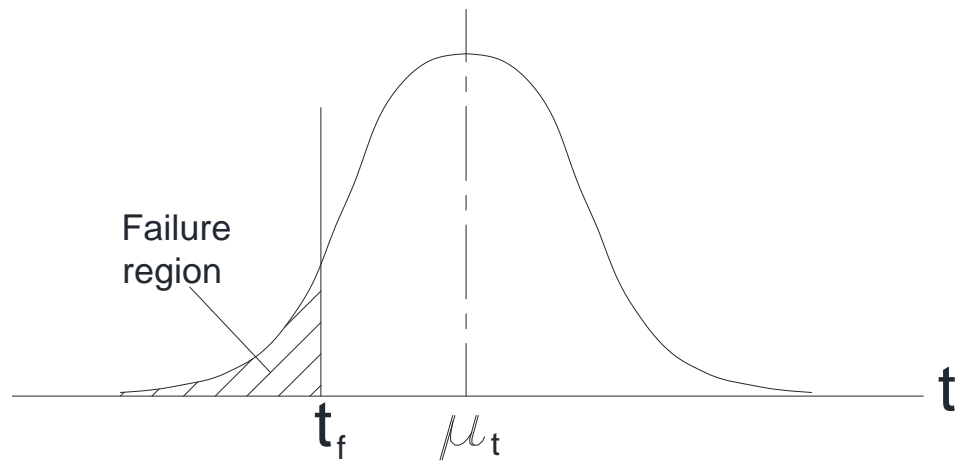

a) Normal

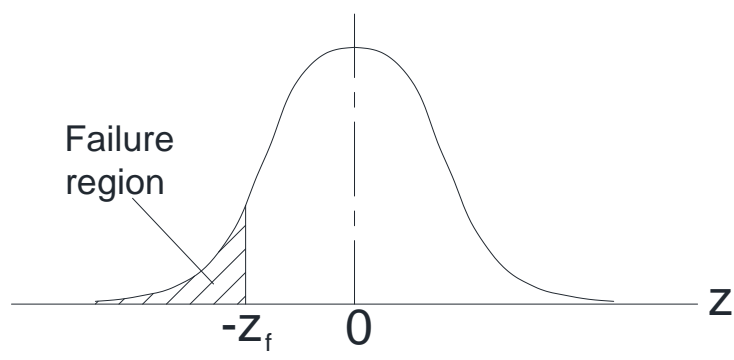

b) Unit normal

Fig. 1: Random variable with normal distribution

The failure zone of a component or product is the region of $t \leq 0$ where $n \leq 1$. Referring to Fig. 1b, any normal variate $t_{f}$ on the left of $\mu_{t}$ in Fig. 1a corresponding to $-z_{f}$ on the left of the origin in Fig. $1 \mathrm{~b}$ can be obtained as:

$$
t_{f}=\mu_{t}-z_{f} s_{t}
$$

Hence when $t_{f}=0$ :

$$
z_{f}=\frac{\mu_{t}}{s_{t}}=\frac{\ln \left(\mu_{n}\right)-0.5 s_{t}^{2}}{s_{t}}
$$

By definition from Eq, (2):

$$
n_{z}=\frac{\mu_{C F}}{\mu_{C M}}
$$

For design application, it may be assumed that $\mu_{n}=n_{z}$ [24, 29] in Eq. (18):

$$
z_{f}=\frac{\ln \left(n_{z}\right)-0.5 s_{t}^{2}}{s_{t}}
$$

Eq. (A11) gives the unit normal variate $z$, based on the reliability factor and design model parameters variability. The modification of $s_{t}$ in Eq. (A7) slightly increases the denominator and simultaneously decreases the numerator of Eq. (A11). The overall effect of this is the reduction of the unit normal variate or $z$-variable, so that the predicted reliability is conservative.

From Eq. (A11):

$$
n_{z}=\exp \left[s_{t}\left(z_{f}+0.5 s_{t}\right)\right]
$$

Eq. (A12) is used to obtain a reliability factor for a desired reliability target corresponding to $z$. The modification of $s_{t}$ in Eq. (A7) slightly increases the reliability factor in Eq. (A12). Hence Eqns. (A11) and (A12) should yield conservative results for $R_{z}$ and $n_{z}$ so that the model may perhaps apply in high reliability situations. The reliability for a known $n_{z}$ is obtained from Eqs. (A11) and (11).

The reliability estimate based on the unit normal variate is commonly read from Tables given the value of the variate. Since table values are discrete, interpolation may be needed occasionally. For use in a spreadsheet, it is more convenient to code a formula. Using data from [5], a curve fitting study was done with the following results. 
For $1.28 \leq z<7.0$ :

$$
R_{z}=1-10^{-\left(0.2006 z^{2}+0.2299 z+0.3818\right)}
$$

Eq. (A13) summarizes the data often presented in tables for the cumulative distribution function of the standard or unit normal probability function for reliability level of at least $90 \%$.

Eqns (A11) and (A12) are remarkable in simplicity because they are functions of only a reliability parameter $(z)$ and variability parameter $\left(s_{t}\right)$. The absolute values of design parameters are not required. Eqn. (A7) combines all the uncertainties in a design task into one parameter: the reliability factor. From Eq. (A12), if the variability of the significant factors in a design model can be estimated with reasonable accuracy, it is possible to design to a reliability target through a reliability factor.

\section{Service Load Factor}

Actual loads on equipment in service vary to different degrees depending on the environment, application, assembling accuracy, and proper installation. They are only known after the product is placed in service and as such are not easy to predict at the design stage. Experience shows that forces acting on equipment in service are generally higher than the rated or nominal values. These higher values are due to mechanical defects and or imperfections in manufacturing, assembling, installations, and operations. Allowed tolerances and inaccuracies in manufacturing, operations, and maintenance activities influence the external and internal dynamics of equipment and so is the misalignment that may arise during assembly and installation. These lead to load increases above rated values so that the design load is often estimated by multiplying the rated load with a service load factor. The service load factor, thus capture the influences of manufacturing, assembly and installation deficiencies and other operating factors influencing the service loads if derived from tests.

In cross-sectional stress design capacity models, the variability of the applied load and stress concentration factor should be considered. Stress concentration arises from geometric changes in component cross-section along its length. Influence of stress concentration is local and must be evaluated section by section. A service load factor for crosssectional stress based design may be expressed as:

$$
\begin{aligned}
& K_{s}=K_{s o} K_{s k} \\
& \vartheta_{s f}=\sqrt{\vartheta_{s o}^{2}+\vartheta_{s k}^{2}+\vartheta_{s a}^{2}+\vartheta_{s m}^{2}}
\end{aligned}
$$

\section{Mechanical Capability Adjustment}

The laboratory test samples for mechanical capabilities like tensile strength, fatigue strength, etc. are very carefully prepared and under closely controlled conditions. Field conditions hardly ever match or correlate exactly with laboratory conditions. For practical applications, modification factors for size, temperature, etc., are applied to laboratory results in a multiplicative capability correction model. For fatigue strength for instance, temperature, size, and surface roughness are very important. More factors may be considered as influencing mechanical capability in specific design situations. When a particular factor is not applicable, then the value is set to unity and the cov is set to zero.

Stress-based design capacity models are of two types: crosssectional and surface stress models. The design examples considered in this paper belong to the cross-sectional stress type. The mechanical capability of a component with a cross-sectional stress design capacity model may be expressed as:

$$
\mu_{C F}=\mu_{C F}^{\prime} C_{f s}
$$

$\mu_{C F}=$ adjusted mechanical capability

$\mu_{C F}^{\prime}=$ nominal mechanical capability

$C_{f s}=$ service capability adjustment factor

$$
\begin{aligned}
& \vartheta_{C F}=\sqrt{\vartheta_{f o}^{2}+\vartheta_{f c}^{2}+\vartheta_{f a}^{2}+\vartheta_{f m}^{2}} \\
& C_{f c}=C_{t} C_{z} C_{r} \\
& \vartheta_{f c}=\sqrt{\vartheta_{c t}^{2}+\vartheta_{c z}^{2}+\vartheta_{c r}^{2}}
\end{aligned}
$$

In fatigue design for example, an adjustment multiplicative model with a correlation coefficient of 0.85 [7] is commonly used. Thus a model variance should be considered when these adjustment factors are used, including the variance of each adjustment factor. Based on the correlation coefficient mentioned, model correlation accuracy cov of 0.05 appears reasonable here. This will be considered as miscellaneous cov for mechanical capability where adjustment factors are applied, since fatigue data generally have more scatter than other modes of failure. That is $\vartheta_{f m}=0.05$. 